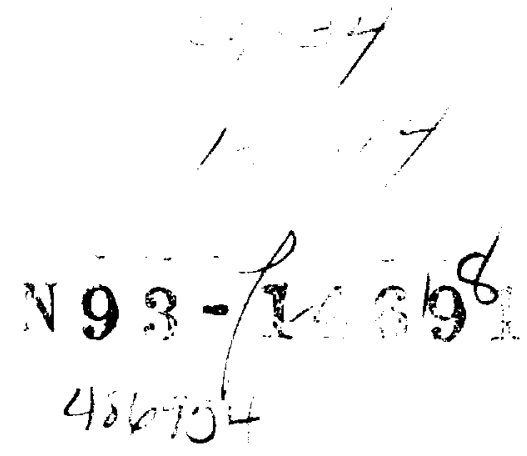

\title{
NUMERICAL SIMULATION OF STEADY AND UNSTEADY ASYMMETRIC VORTICAL FLOW
}

\author{
O. A. Kandil and T.-C. Wong \\ Department of Mechanical Engineering and Mechanics, Old Dominion University \\ Norfolk, VA 23529, U.S.A. \\ AND \\ C. H. LiU \\ Theoretical Flow Physics Branch, NASA Langley Research Center, \\ Hampton, VA 23665, U.S.A.
}

\section{Reprinted from}

Journal of Fluids and Structures (1992) 6, 249-265 


\title{
NUMERICAL SIMULATION OF STEADY AND UNSTEADY ASYMMETRIC VORTICAL FLOW
}

\author{
O. A. Kandil AND T.-C. Wong \\ Department of Mechanical Engineering and Mechanics, Old Dominion University \\ Norfolk, VA 23529, U.S.A. \\ AND \\ C. H. LiU \\ Theoretical Flow Physics Branch, NASA Langley Research Center, \\ Hampton, VA 23665, U.S.A.
}

(Received 7 August 1990 and in revised form 1 August 1991)

The unsteady, compressible, thin-layer, Navier-Stokes (NS) equations are solved to simulate steady and unsteady, asymmetric, vortical laminar flow around cones at high incidences and supersonic Mach numbers. The equations are solved by using an implicit, upwind, flux-difference splitting (FDS), finite-volume scheme. The locally conical flow assumption is used and the solutions are obtained by forcing the conserved components of the flowfield vector to be equal at two axial stations located at 0.95 and 1.0 . Computational. examples cover steady and unsteady asymmetric flows around a circular cone and its control using side strakes. The unsteady asymmetric flow solution around the circular cone has also been validated using the upwind, flux-vector splitting (FVS) scheme with the thin-layer NS equations and the upwind FDS with the full NS equations. The results are in excellent agreement with each other. Unsteady asymmetric flows are also presented for elliptic- and diamond-section cones, which model asymmetric vortex shedding around round- and sharp-edged delta wings.

\section{INTRODUCTION}

AT HIGH ANGLES OF ATTACK, flow separations from the forebodies of missiles and fighter aircraft may become asymmetric resulting in side forces, yawing moments and rolling moments which are, in many instances, sufficiently large to trigger missile and aircraft spin. Experimental studies have shown that it is not necessary for the separation lines to be asymmetric in order for the separated flow to be asymmetric (Kenner \& Chapman 1977; Peak et al. 1979; Lamont 1980, 1982). These studies have also shown that unsteady asymmetric flow with vortex shedding may be either random or periodic, where the latter is similar to the Kármán vortex street in two-dimensional flows around cylinders.

The onset of flow asymmetry occurs when the relative incidence (ratio of angle of attack to semi-apex angle) of pointed forebodies exceeds certain critical values. At the critical values of relative incidence, flow asymmetry develops due to natural and/or forced disturbances. The origin of natural disturbances may be a transient side slip, an acoustic disturbance, or similar disturbance of short duration. The origin of forced disturbances is geometric perturbations due to imperfections in the nose geometric symmetry or similar disturbances of permanent nature. In addition to the relative incidence as one of the determinable parameters for the onset of flow asymmetry, the free-stream Mach number, Reynolds number and shape of the body cross-sectional area are important determinable parameters. Asymmetric flow and vortex shedding 
have also been documented for sharp-edged delta wings at very high relative incidences (Shanks 1963; Rediniotis et al. 1988).

The mechanisms which lead to steady and unsteady asymmetric vortical flows past wings and bodies at high angles of attack and zero side slip are not well understood. The experimental studies of these phenomena by several investigators (e.g. Keener \& Chapman 1977; Lamont 1982; Skow \& Peake 1982; Peake \& Tobak 1982) propose two mechanisms for explaining the origin of flow asymmetry. The first mechanism suggests that the asymmetry occurs due to instability of the velocity profiles in the vicinity of the enclosing saddle point which exists in the cross-flow planes above the body primary vortices. The second mechanism suggests that the asymmetry occurs due to asymmetric transition of the boundary-layer flow at the apex, either in the axial direction or on both sides of the body in the cross-flow plane.

Very recently, several attempts have been carried out to computationally simulate asymmetric vortical flows around slender bodies of revolution. In a paper by Marconi (1988), the Euler equations are used along with a "forced separation model", which is introduced by Fiddes (1989), to solve for supersonic flow past a circular cone. The pseudo-time stepping is carried out until the residual error reaches machine zero while the flow is symmetric. Proceeding with the time stepping, vortex-flow asymmetry is obtained and stays stable thereafter. It is believed that the asymmetry is triggered by the machine round-off error, which acts as a disturbance to the saddle point in the flowfield. This work shows that the first mechanism of asymmetric vortex flow is basically an inviscid mechanism. In a recent paper by Siclari \& Marconi (1989), the full Navier-Stokes equations are used to solve for supersonic asymmetric flows around a $5^{\circ}$-semi-apex angle cone over a wide range of angles of attack.

Kandil et al. (1990a) used the unsteady, thin-layer Navier-Stokes equations along with two different implicit schemes to simulate asymmetric vortex flows around cones with different cross-sectional shapes. The numerical investigation focuses on a $5^{\circ}$-semi-apex angle circular cone and locally conical flow is assumed. The first scheme is an implicit, upwind, flux-difference splitting, finite-volume scheme and the second one is an implicit, approximately factored central-difference, finite-volume scheme. Keeping the Mach number and Reynolds number constant at 1.8 and $10^{5}$, respectively, the angle of attack is varied from 10 to $30^{\circ}$. At $\alpha=10^{\circ}$, a steady symmetric solution is obtained and the results of the two schemes are in excellent agreement. At $\alpha=20^{\circ}$ and irrespective of the type or level of the disturbance, a unique steady asymmetric solution is obtained and the results of the two schemes are in excellent agreement. Two types of flow disturbances are used: a random round-off error or a random truncation-error disturbance, and a controlled transient side-slip disturbance with short duration. For the controlled transient side-slip disturbance the solution is unique, and for the uncontrolled random disturbance the solution is also unique with the exception of having the same asymmetry changing sides on the cone. At $\alpha=30^{\circ}$, an unsteady asymmetric solution with vortex shedding is obtained, and the vortex shedding is perfectly periodic. Next, the angle of attack is kept fixed at $20^{\circ}$ and the Mach number is increased from 1.8 to 3.0 with a step of 0.4 . The solutions show that the asymmetry becomes weaker as the Mach number is increased. The flow recovers its symmetry when the Mach number reaches 3.0. Passive control of the flow asymmetry has also been tentatively demonstrated by using a fin on the leeward side of the body along the plane of geometric symmetry.

Experimental research efforts have also been directed to control asymmetric flows for eliminating or attenuating the asymmetric forces and the resulting moments by using either passive-control or active-control methods. Passive-control methods include 
the use of a vertical fin on the leeward side along the plane of geometric symmetry (Stahl 1989), the use of fixed or movable forebody strakes (Skow \& Peak 1982; Ng 1989 ), or the use of a rotatable forebody tip having variable cross-section, from a circular shape at its base to an elliptic shape at its tip (Moskovitz et al. 1990). Active-control methods primarily include the use of blowing ports with various blowing rates and directions on the forebody surface $(\mathrm{Ng} 1990)$. Computational simulations have also been used to study the effectiveness of both passive (Kandil et al. 1990a) and active control methods (Travella et al. 1990).

In this paper, the unsteady, compressible, laminar, thin-layer, Navier-Stokes equations are used, along with an implicit, upwind, flux-difference splitting, finitevolume scheme to solve for steady and unsteady, asymmetric vortex flows around cones. The steady results include asymmetric flow around a circular cone and its control using side strakes. The unsteady results include the asymmetric vortex shedding around circular, elliptic- and diamond-section cones. The unsteady results for the circular cone are verified by using the FVS with the thin-layer Navier-Stokes equations and the FDS with the full Navier-Stokes equations.

\section{FORMULATION}

\subsection{Governing Eouations}

The three-dimensional compressible viscous flow around the body is governed by the conservative form of the dimensionless, unsteady, compressible, thin-layer NavierStokes equations. In terms of time-independent body-conformed coordinates $\xi^{1}, \xi^{2}$ and $\xi^{3}$, the equations are

$$
\frac{\partial Q}{\partial t}+\frac{\partial E_{s}}{\partial \xi^{s}}-\frac{\partial\left(E_{v}\right)}{\partial \xi^{3}}=0
$$

where

$$
\begin{gathered}
Q=\frac{\tilde{q}}{J}=\frac{1}{J}\left[\rho, \rho u_{1}, \rho u_{2}, \rho u_{3}, \rho \mathrm{e}\right]^{t} ; \\
E_{m} \equiv \text { inviscid flux }=\frac{1}{J}\left[\partial_{k} \xi^{m} \hat{E}_{k}\right]^{t} \\
=\frac{1}{J}\left[\rho U_{m}, \rho u_{1} U_{m}+\partial_{1} \xi^{m} p, \rho u_{2} U_{m}+\partial_{2} \xi^{m} p, \rho u_{3} U_{m}\right. \\
\left.+\partial_{3} \xi^{m} p,(\rho e+p) U_{m}\right]^{t}, m=1,2,3 ; \\
\left(E_{v}\right)_{3} \equiv \operatorname{viscous} \text { and heat-conduction flux in } \xi^{3} \text { direction } \\
=\frac{1}{J}\left[0, \partial_{k} \xi^{3} \tau_{k 1}, \partial_{k} \xi^{3} \tau_{k 2}, \partial_{k} \xi^{3} \tau_{k 3}, \partial_{k} \xi^{3}\left(u_{n} \tau_{k n}-q_{k}\right)\right]^{z} ; \\
U_{m}=\partial_{k} \xi^{m} u_{k} .
\end{gathered}
$$

The first element of the three momentum elements of equation (4) is given by

$$
\partial_{k} \xi^{3} \tau_{k 1}=\frac{\mathbf{M}_{\infty} \mu}{\operatorname{Re}}\left(\psi \partial_{1} \xi^{3}+\frac{\partial u_{1}}{\partial \xi^{3}}\right)
$$


where

$$
\phi=\partial_{k} \xi^{3} \partial_{k} \xi^{3}, \quad \psi=\frac{1}{3} \partial_{k} \xi^{3} \frac{\partial u_{k}}{\partial \xi^{3}}
$$

The second and third elements of the momentum elements are obtained by replacing the subscript 1 , everywhere in equation (6), with 2 and 3 , respectively. The last element of equation (4) is given by

$$
\partial_{k} \xi^{3}\left(u_{n} \tau_{k n}-q_{k}\right) \equiv \frac{\mathbf{M}_{\infty} \mu}{\operatorname{Re}}\left\{\psi W+\phi\left[\frac{1}{2} \frac{\partial}{\partial \xi^{3}} u_{s} u_{s}+\frac{1}{(\gamma-1) \operatorname{Pr}} \frac{\partial\left(a^{2}\right)}{\partial \xi^{3}}\right]\right\}
$$

where

$$
W=\partial_{n} \xi^{3} u_{n}
$$

The reference parameters for the dimensionless form of the equations are $L, a_{\infty}, L / a_{\infty}$, $\rho_{\infty}$ and $\mu_{\infty}$ for the length, velocity, time, density and molecular viscosity, respectively. The Reynolds number is defined as $\operatorname{Re}=\rho_{\infty} V_{\infty} L / \mu_{\infty}$, and the pressure, $p$, is related to the total energy per unit mass, $e$, and density, $\rho$, by the gas equation

$$
p=(\gamma-1) \rho\left(\mathrm{e}-\frac{1}{2} u_{n} u_{n}\right) \text {. }
$$

The viscosity, $\mu$, is calculated from the Sutherland law

$$
\mu=T^{3 / 2}\left(\frac{1+C}{T+C}\right), \quad C=0.4317,
$$

and the Prandtl number $\operatorname{Pr}=0.72$.

In equations (1)-(10), the indicial notation is used for convenience. The subscripts $k$ and $n$ are summation indices, the superscript or subscript $s$ is a summation index and the superscript or subscript $m$ is a free index. The range of $k, n, s$ and $m$ is $1-3$, and $\partial_{k} \equiv \partial / \partial x_{k}$. In equations (1)-(11), $u_{n}$ is the Cartesian velocity component, $U_{m}$ the contravariant velocity component, $\tau_{m}$ the Cartesian component of the shear stress tensor, $q_{k}$ the Cartesian component of heat flux vector, $a$ the local speed of sound and $\mathrm{M}_{\infty}$ the free-stream Mach number.

\subsection{Boundary and InItIal Conditions}

Boundary conditions are explicitly implemented. They include inflow-outflow conditions and solid-boundary conditions. At the plane of geometric symmetry, periodic conditions are used for symmetric or asymmetric applications on the whole computational domain (right and left domains). Since we are dealing with supersonic flows, at the far-field inflow boundaries, free-stream conditions are specified, and the conical shock is captured as part of the solution. At the far-field outflow boundaries first-order extrapolation from the interior points is used. On the solid boundary, the no-slip and no-penetration conditions are enforced, $u_{1}=u_{2}=u_{3}=0$, and the normal pressure gradient is set equal to zero. For the temperature, the adiabatic boundary condition is enforced on the solid boundary. The initial conditions are set equal to the free-stream conditions with $u_{1}=u_{2}=u_{3}=0$ on the solid boundary.

For the passive control application using side strakes, solid-boundary conditions are enforced on both sides of the strake.

\section{COMPUTATIONAL SCHEMES}

The principal computational scheme used to solve the governing equations is an implicit, upwind, flux-difference splitting, finite-volume scheme. It employs the 
flux-difference splitting scheme of Roe. The Jacobian matrices of the inviscid flux-differences are split into left and right flux differences according to the signs of the eigenvalues of the inviscid Jacobian matrices. The smooth flux limiters are used to eliminate oscillations in the shock region. The viscous and heat-flux terms are centrally differenced. The resulting difference equation is solved using approximate factorization along the $\xi^{1}, \xi^{2}$ and $\xi^{3}$ directions, respectively. The scheme is third-order accurate in space and first-order accurate in time. The computational scheme is coded in the computer program "CFL3D." Details of the scheme are given by Rumsey \& Anderson (1988).

The second scheme is an implicit, approximately factored, central-difference, finite-volume scheme. Added second-order and fourth-order dissipation terms are used in the difference equation on its right-hand side terms, which represent the explicit part of the scheme. The Jacobian matrices of the implicit operator on the left-hand side of the difference equation are centrally differenced in space, and implicit second-order dissipation terms are added for the numerical stability. The left-hand side operator is approximately factored, and the difference equation is solved in three sweeps along the $\xi^{1}, \xi^{2}$ and $\xi^{3}$ directions, respectively. The computational scheme is coded in the computer program "ICF3D". Details of the scheme are given by Kandil \& Chuang (1989). The ICF3D code is used to verify some cases which are solved by the CFL3D code. In this paper, the ICF3D code is used to validate the steady asymmetric flow case.

A third computational scheme is used to validate the unsteady asymmetric vortex flow around the circular cone at a $30^{\circ}$ angle of attack. This scheme is the flux-vector splitting scheme which is based on the van Leer flux-vector splitting scheme (Rumsey \& Anderson 1988). This optional scheme is also coded in the computer program "CFL3D".

Since the applications in this paper cover locally conical flows only, the threedimensional codes are used to solve for locally conical flows at the axial station $x_{1}=1$. This is achieved by forcing the conserved components of the flowfield vector, $\hat{q}$, to be equal at two planes located at $x_{1}=0.95$ and $1 \cdot 0$. The concept of locally conical flow is explained in the next section.

\section{LOCALLY CONICAL FLOWS}

Locally conical solutions of the thin-layer or full Navier-Stokes equations are obtained using one of two methods. In the first method, the governing equations are transformed using the conical-coordinate transformation. Invoking the conical flow condition which requires that the flow variables be independent of the radial distance (or axial distance, depending on the transformation) from the cone apex, equating the radial distance (or axial distance) which appears in the transformed equations to a constant (equals to unity in most of the present locally conical solutions), the resulting equations are solved on one spherical (or cross-flow) surface. In the second method, the three-dimensional flow equations are solved on two spherical (or cross-flow) surfaces which are located in the very near proximity of a constant radial (or axial) distance.

During the pseudo-time or accurate-time stepping, the flowfield vector is forced to be equal at the corresponding grid centers on the two surfaces. This method is used in the present paper to obtain locally conical solutions. The resulting solutions from these two methods are the same locally conical solutions. These solutions correspond to the specified radial (or axial) distance and hence they change as the radial (or axial) 
distance is changed. The reason behind that is simply because the transformed equations, according to the first method, are not self-similar and hence they are not globally conical. This is shown below by developing the transformed equations of the first method. Considering the unsteady, compressible, Navier-Stokes equations in the Cartesian coordinates,

$$
\frac{\partial q}{\partial t}+\frac{\partial\left(\mathbf{E}-\mathbf{E}_{v}\right)_{i}}{\partial x_{i}}=0, \quad i=1-3
$$

introducing the conical coordinates,

$$
\eta_{1}=\frac{x_{1}}{x_{3}}, \quad \eta_{2}=\frac{x_{2}}{x_{3}}, \quad \eta_{3}^{2}=x_{i} x_{i}
$$

and using the chain rule, equation (12) is transformed to

$$
\frac{\eta_{3}}{m} \frac{\partial q}{\partial t}+\frac{\partial}{\partial \eta_{1}}\left(\overline{\mathbf{E}}-\overline{\mathbf{E}}_{v}\right)_{1}+\frac{\partial}{\partial \eta_{2}}\left(\overline{\mathbf{E}}-\overline{\mathbf{E}}_{v}\right)_{2}+\frac{\eta_{3}}{m^{2}} \frac{\partial}{\partial \eta_{3}}\left(\overline{\mathbf{E}}-\overline{\mathbf{E}}_{v}\right)_{3}+2\left(\overline{\mathbf{I}}-\overline{\mathbf{L}}_{v}\right)=0,
$$

where

$$
\begin{aligned}
m & =\sqrt{1+\eta_{1}^{2}+\eta_{2}^{2}}, \quad \overline{\mathbf{E}}_{1}=\mathbf{E}_{1}-\eta_{1} \mathbf{E}_{3}, \\
\overline{\mathbf{E}}_{2} & =\mathbf{E}-\eta_{2} \mathbf{E}_{3}, \quad \overline{\mathbf{E}}_{3}=\mathbf{E}_{3}+\eta_{1} \mathbf{E}_{1}+\eta_{2} \mathbf{E}_{2}, \\
\overline{\mathbf{I}} & =\overline{\mathbf{E}}_{3}, \quad \overline{\mathbf{E}}_{v 1}=\mathbf{E}_{v 1}-\eta_{1} \mathbf{E}_{v 3}, \\
\overline{\mathbf{E}}_{v 2} & =\mathbf{E}_{v}-\eta_{2} \mathbf{E}_{v 3}, \quad \overline{\mathbf{E}}_{v 3}=\mathbf{E}_{v 3}+\eta_{1} \mathbf{E}_{v 1}+\eta_{2} \mathbf{E}_{v 2}, \\
\overline{\mathbf{i}}_{v} & =\overline{\mathbf{E}}_{v 3} .
\end{aligned}
$$

The conical flow condition requires that the flow variables be independent of the coordinate $\eta_{3}$ (radial distance). Invoking this condition in equation (14) by dropping the derivatives with respect to $\eta_{3}$, equation (14) reduces to

$$
\frac{\eta_{3}}{m} \frac{\partial q}{\partial t}+\frac{\partial}{\partial \eta_{1}}\left(\overline{\mathbf{E}}-\overline{\mathbf{E}}_{v}\right)_{1}+\frac{\partial}{\partial \eta_{2}}\left(\overline{\mathbf{E}}-\overline{\mathbf{E}}_{v}\right)_{2}+2\left(\overline{\mathbf{I}}-\overline{\mathbf{L}}_{v}\right)=0
$$

It is obvious that the unsteady term includes $\eta_{3}$. Moreover, the viscous terms $\partial \overline{\mathbf{E}}_{v 1} / \partial \eta_{1}, \partial \overline{\mathbf{E}}_{v 2} / \partial \eta_{2}$ and $\overline{\mathbf{L}}_{v}$ include $\eta_{3}$, and hence equation (19) is not self-similar. The explicit dependence of the viscous terms on $\eta_{3}$ can be shown through one of the elements of these vectors. For example, we consider

$$
\begin{array}{r}
\frac{\partial}{\partial \eta_{1}}\left(\tau_{x x}-\eta_{1} \tau_{x z}\right)=\frac{\mathbf{M}_{\infty}}{R_{3}} \frac{\partial}{\partial \eta_{1}}\left[\mu\left(2 \frac{\partial u_{1}}{\partial x}-\frac{2}{3}\left(\frac{\partial u_{1}}{\partial x}+\frac{\partial u_{2}}{\partial y}+\frac{\partial u_{3}}{\partial z}\right)-\eta_{1}\left(\frac{\partial u_{1}}{\partial z}+\frac{\partial u_{3}}{\partial x}\right)\right)\right] \\
=\frac{\mathbf{M}_{\infty} m}{\operatorname{Re} \eta_{3}} \frac{\partial}{\partial \eta_{1}}\left[\mu\left(\left(\frac{4}{3}-\eta_{1}^{2}\right) \frac{\partial u_{1}}{\partial \eta_{1}}+\eta_{1} \frac{\partial u_{1}}{\partial \eta_{2}}-\frac{2}{3} \frac{\partial u_{2}}{\partial \eta_{2}}-\frac{\eta_{1}}{3} \frac{\partial u_{3}}{\partial \eta_{1}}+\frac{2}{3} \eta_{2} \frac{\partial u_{3}}{\partial \eta_{2}}\right)\right] .
\end{array}
$$

Thus, the unsteady term and viscous terms are scaled by the radial distance $\eta_{3}$ and equation (16) does not represent a globally conical flow. The best to be done to make use of this equation is to select a constant value for $\eta_{3}$, and solve the resulting equation for what we call "locally conical flow". If $\eta_{3}$ is assigned another constant value, the resulting equation will have another scale for the unsteady term and viscous terms. It is concluded that equation (16) becomes globally conical if the unsteady term and viscous terms vanish, and hence only the steady Euler equations are globally conical. 


\section{COMPUTATIONAL APPLICATIONS}

\subsection{Steady Asymmetric Flow Around a Circular Cone}

A grid of $161 \times 81$ points in the circumferential and normal directions, respectively is used. The grid is generated by using a modified Joukowski transformation with a geometric series for the grid clustering near the solid boundary. The minimum grid spacing at the solid boundary in the normal direction is $10^{-4}$ (the characteristic length is the conical station at $x_{1}=1$ ). A $5^{\circ}$-semi-apex angle cone at angle of attack, $\alpha$, of $20^{\circ}$, free-stream Mach number, $M_{\infty}$, of 1.8 and Reynolds number, Re, of $10^{5}$ is used. The maximum radius of the computational domain is $21 r$, where $r$ is the cone cross-section radius at the axial station $x_{1}=1$.

Figure 1 shows the residual error versus the number of iterations, the surfacepressure coefficient, the cross-flow velocity and the total-pressure-loss contours for the CFL3D and ICF3D codes. In the residual error figure, the CFL3D code shows that the residual error drops ten orders of magnitude, to machine zero, within 2,500 iteration steps and the solution is still symmetric. Thereafter, the error increases by six-orders of magnitude and slightly asymmetric solutions are obtained. The flow is symmetric during the first 5,000 iteration steps. Next, the error drops down by another six orders of magnitude, to machine zero again, and stays constant, and a stable steady asymmetric solution is obtained. It should be noted here that when the residual error first reaches machine zero, the machine-round-off error acts as a random asymmetric disturbance to the critically symmetric solution. Thereafter, the disturbance grows spatially, producing the asymmetric solution. The ICF3D code shows that the residual error drops five orders of magnitude in the first 3,000 iteration steps, increases two orders of magnitude in the next 2,000 iteration steps, and then drops down by three orders of magnitude within the next 5,000 iterations. The flow solution goes through a
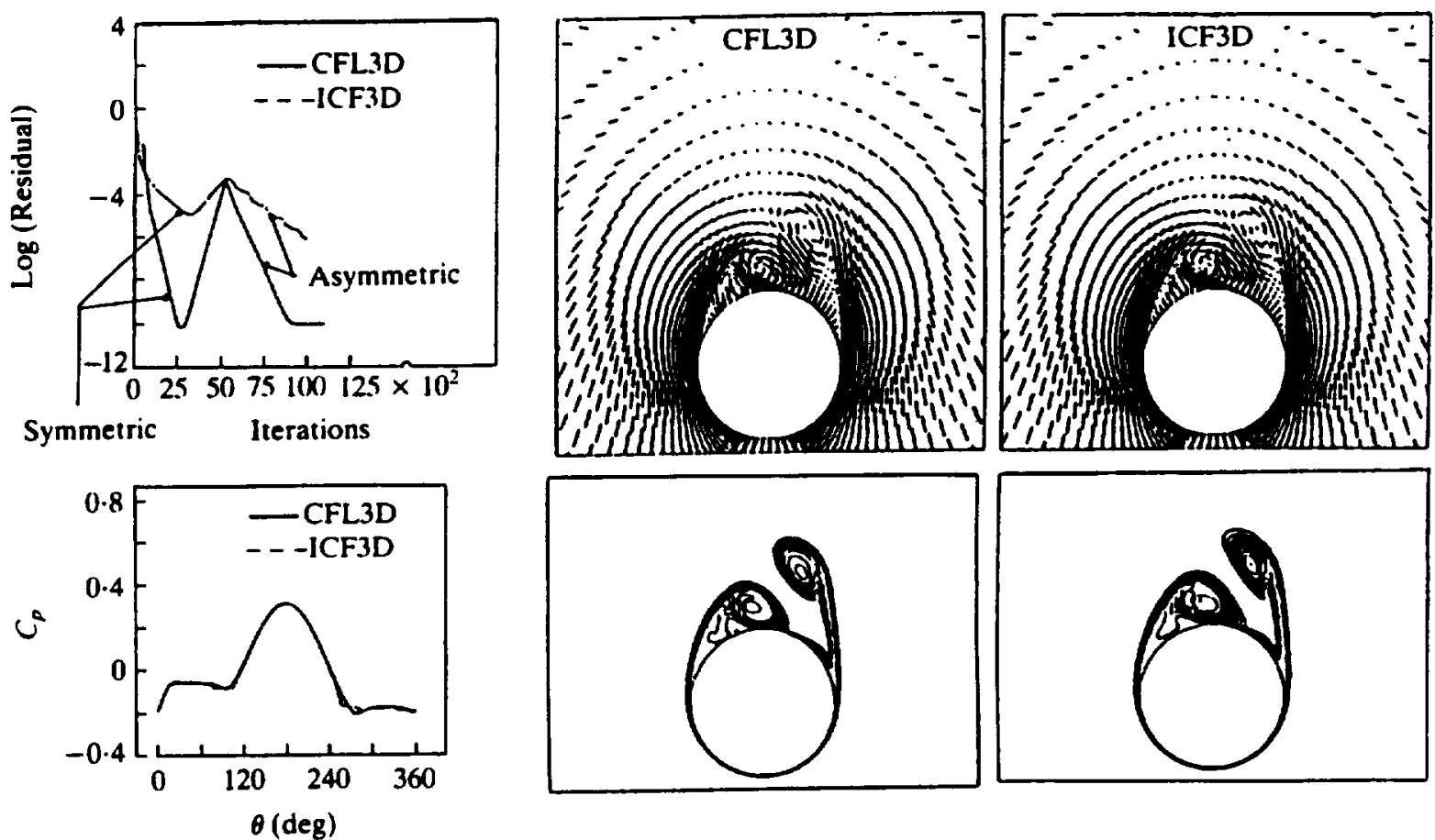

Figure 1. Comparison of steady asymmetric flow solutions around a circular cone, $\alpha=20^{\circ}, \mathrm{M}_{\infty}=1 \cdot 8$, $\operatorname{Re}=10^{5}$. 
symmetric unstable solution and then to the asymmetric stable solution. The pressure coefficient curves for the two codes are in excellent agreement over the circumferential angle $\theta$, which is measured from the leeward plane of symmetry. The suction pressure in the range of $\theta=0-90^{\circ}$ is lower than that in the range of $\theta=270-360^{\circ}$. The cross-flow velocity and total-pressure-loss contours for the two codes are in excellent agreement. They show the nature of the flow asymmetry and its details.

Since the residual error of the CFL3D code is much smaller than that of the ICF3D code, the disturbance which triggered the asymmetry in the first code is attributed to the machine round-off error, while the disturbance which triggered the asymmetry in the second code is attributed to the truncation error of the scheme. Both disturbances are random in nature. However, irrespective of the source of disturbance, the final asymmetric stable solution is unique. Kandil et al. (1990a) have shown that the solution is still unique if another source of disturbance is applied for the same critical flow
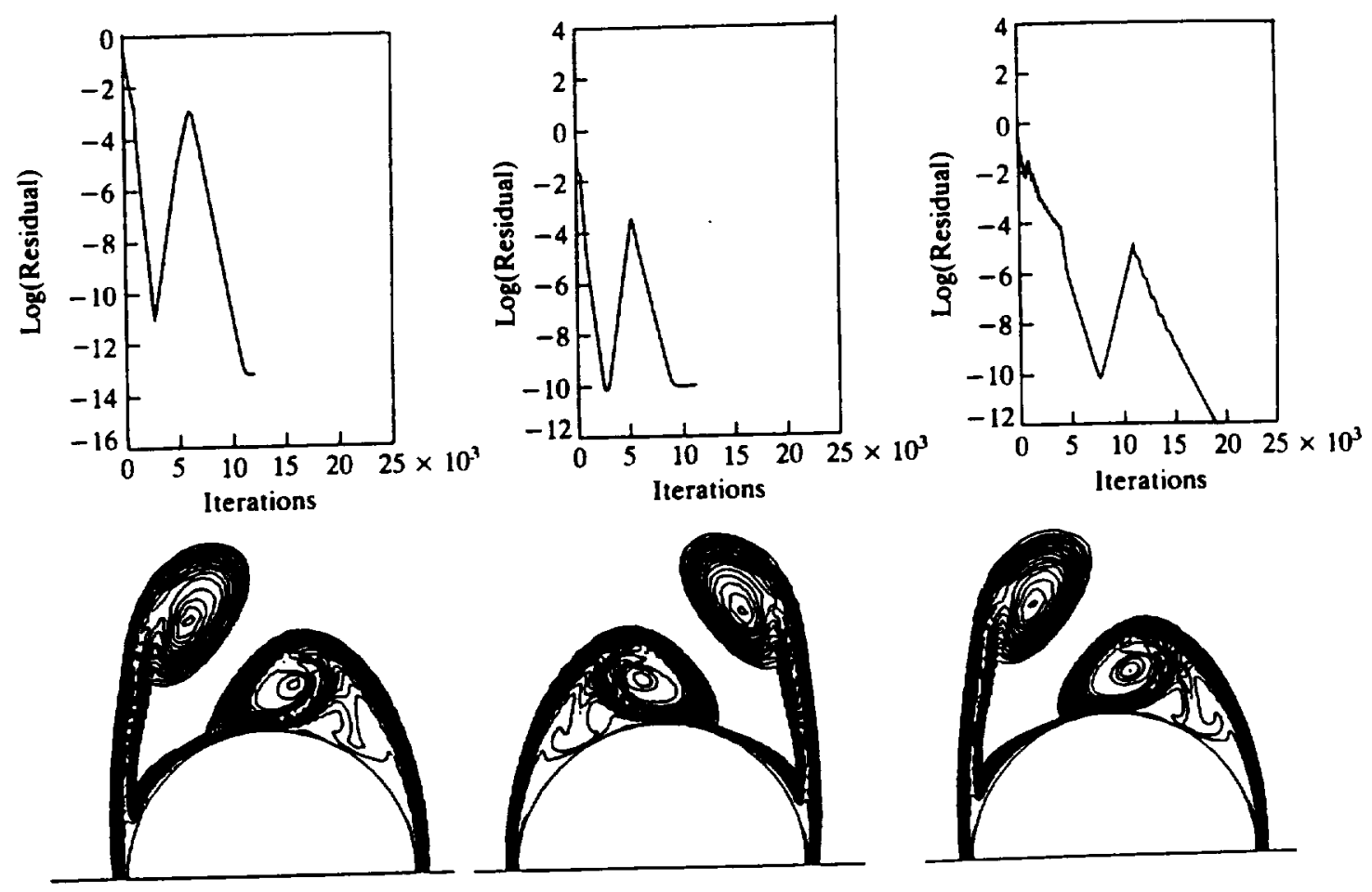

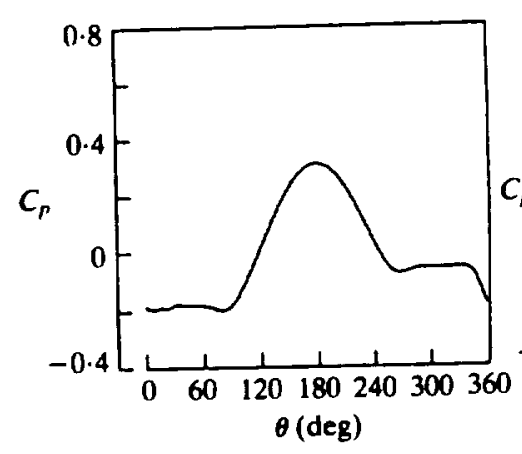

(a) $\Delta \xi_{\min }^{3}=10^{-3}$

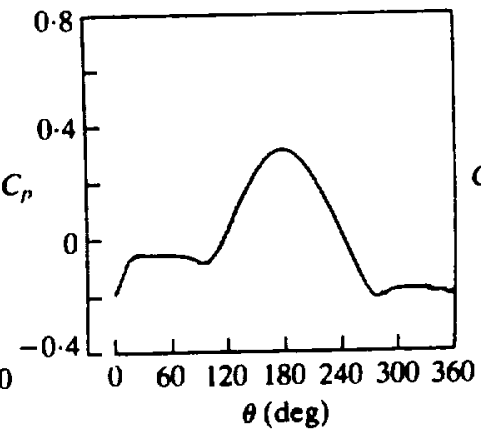

(b) $\Delta \xi_{\min }^{3}=10^{-4}$

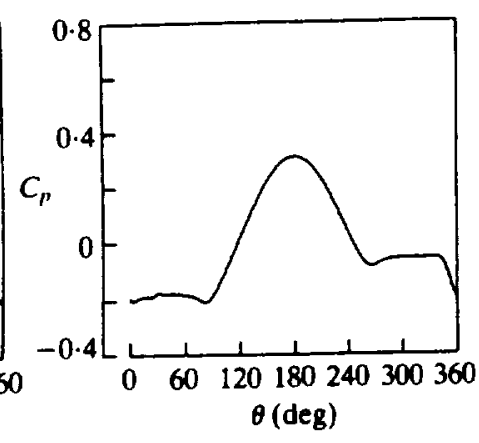

(c) $\Delta \xi_{\min }^{3}==10^{-5}$

Figire 2. Effect of minimum grid size on the asymmetric flow solution; $\alpha=20^{\circ}, M_{\infty}=1 \cdot 8, \operatorname{Re}=10^{5}$. 
conditions; e.g., a $2^{\circ}$ or $0.5^{\circ}$ short-duration side-slip disturbance produces the same flow asymmetry.

In Figure 2, we show the effect of the minimum grid size on the flow asymmetry for the same flow conditions of the previous case. The figure shows the residual error versus the number of iterations, the total-pressure-loss contours and the surfacepressure coefficient for $\Delta \xi_{\min }^{3}=10^{-3}, 10^{-4}$ and $10^{-5}$ at the cone surface. The histories of the residual errors are qualitatively of similar behavior. The total-pressure-loss contours show unique solutions with the exception of having the asymmetry changing sides. This is understood due to the random nature of the disturbance-a machine round-off error. The surface-pressure-coefficient curves also show unique solutions.

With all the numerical experiments given above, it is conclusively proven that the asymmetric solution is not scheme-, numerics- or disturbance-dependent.

\subsection{Passive Control of Asymmetric Flow Using Side Strakes}

Figure 3 shows the results of passive control of flow asymmetry around the circular cone of Figure 1 by using side strakes of height equal to $0.3 r$. The iteration histories of the residual error, lift coefficient and side-force coefficient show the attenuation of the flow asymmetry and the final stable symmetric solution. The surface-pressurecoefficient curve, the cross-flow velocity and the total-pressure-losses contours show the final symmetric solution and the symmetric vortices associated with this controlled flow.
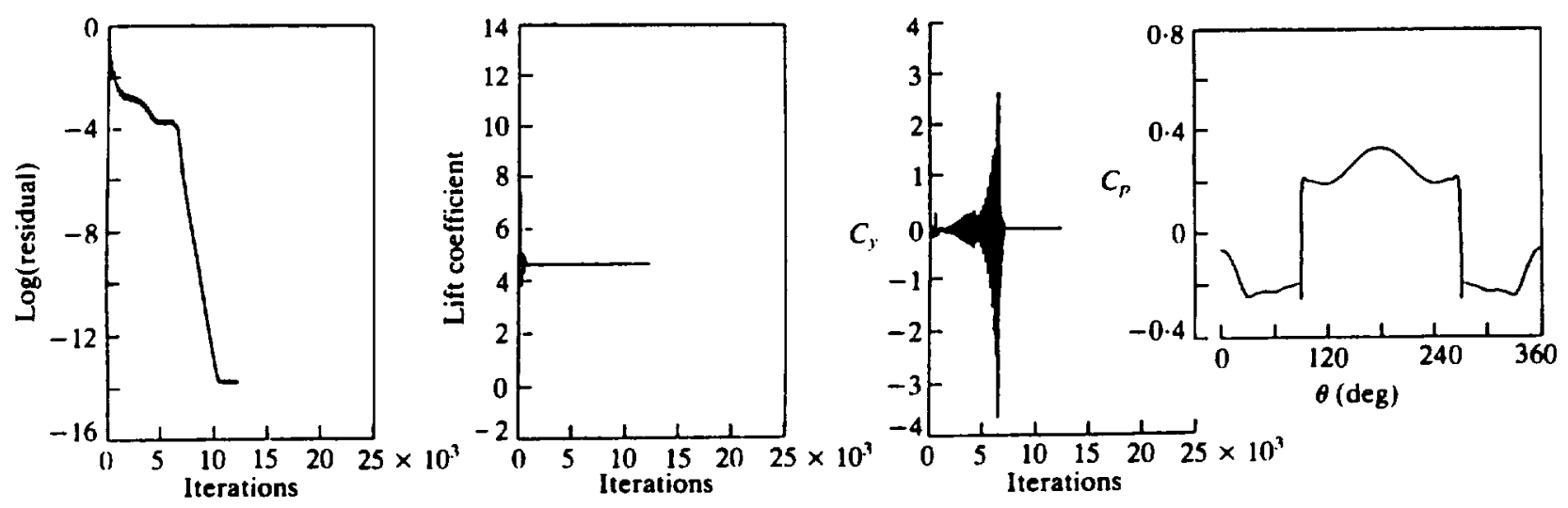

$-0 \cdot 1500,0 \cdot 1800 \quad 0.1500,0 \cdot 18$
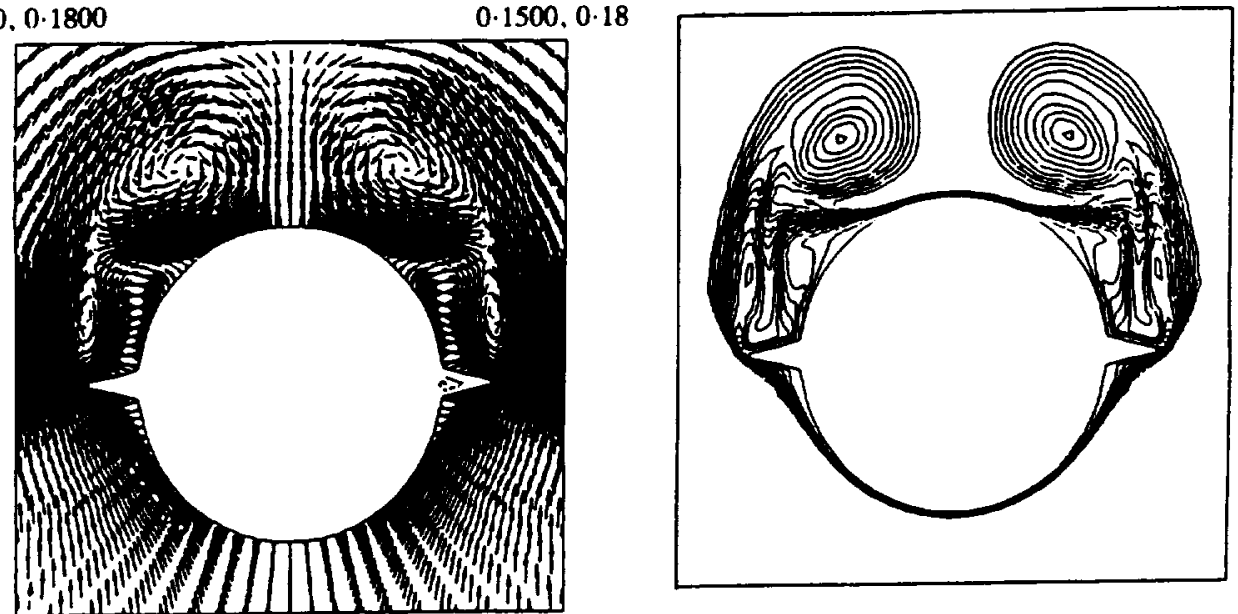

$-0.1500,-0.1200$

$0.1500,-0.1200$

Figure 3. Passive control of asymmetric flow around a circular cone using strakes, $\alpha=20^{\circ}, \mathrm{M}_{\infty}=1.8$, $\operatorname{Re}=10^{5}, h=0.3 r$ ( $h$ and $r$ are local strake height and cone radius, respectively.). 
The side strakes push the primary vortices away from the leeward plane of geometric symmetry, and hence they prevent communication of the flow disturbance from the two sides. It should also be noticed that the $C_{p}$ curves of Figures 1 and 3 show that the side strakes provide additional lift besides their function of controlling the flow asymmetry.

\subsection{Unsteady Asymmetric Flow Around a Circular Cone}

Keeping the Mach number at 1.8 and Reynolds number at $10^{5}$, the angle of attack is increased to $30^{\circ}$ for the flow around the circular cone of Figure 1. Figure 4 shows the results of this case using the FDS scheme with the thin-layer NS equations, FVS-scheme with the thin-layer NS equations and the FDS scheme with the full NS equations. Here, we show the history of the residual error, the lift coefficient and the total-pressure-loss contours. For the first solution, pseudo-time stepping was used up to

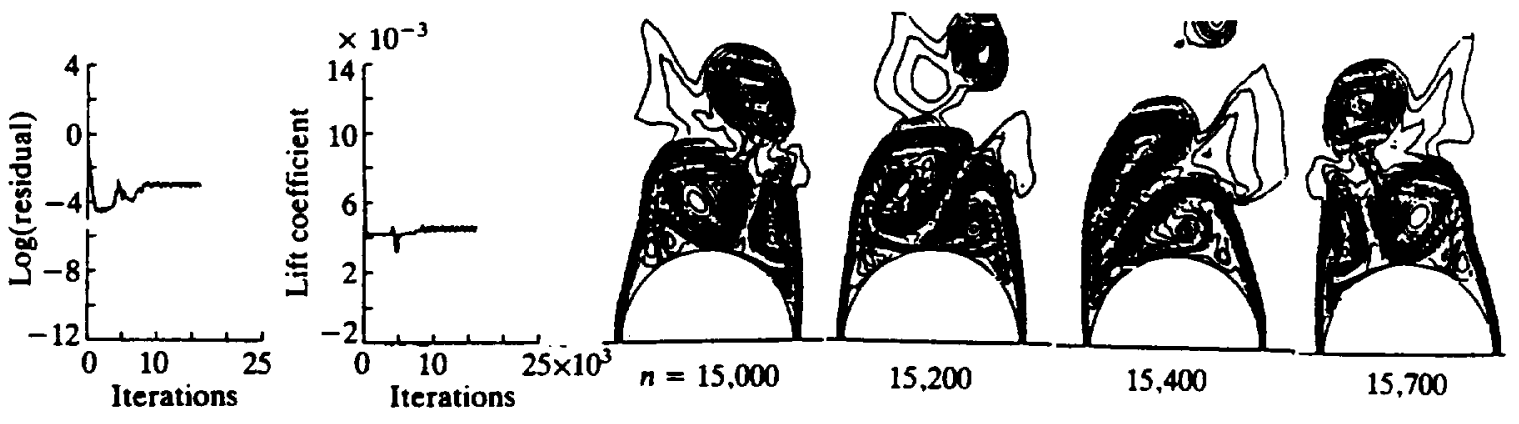

Thin-layer eqs. (FDS)

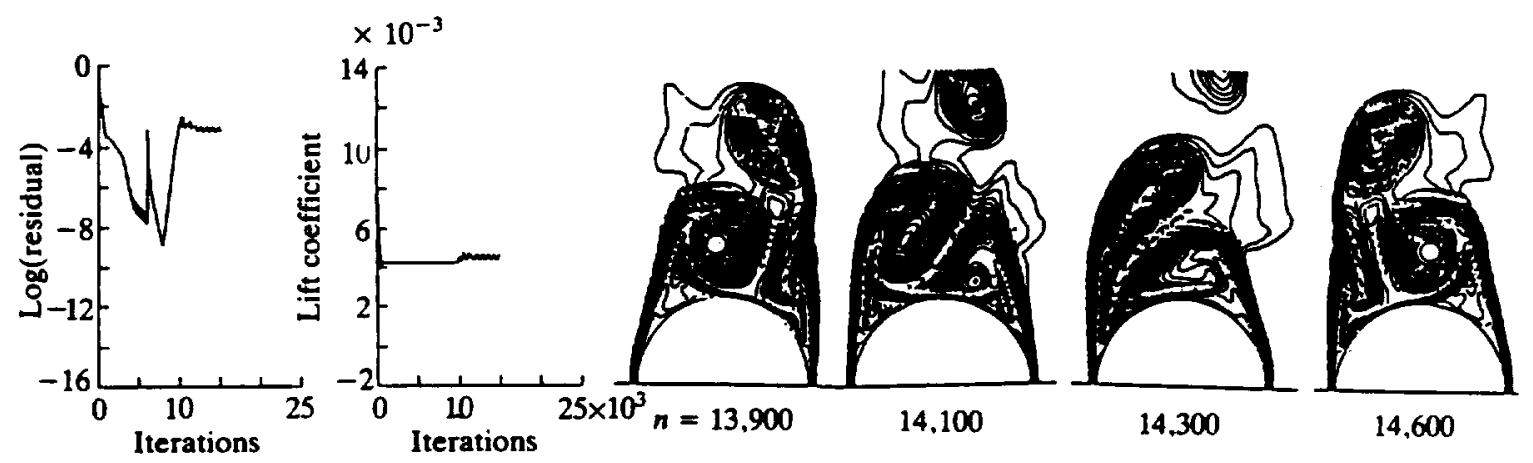

Thin-layer eqs. (FVS)
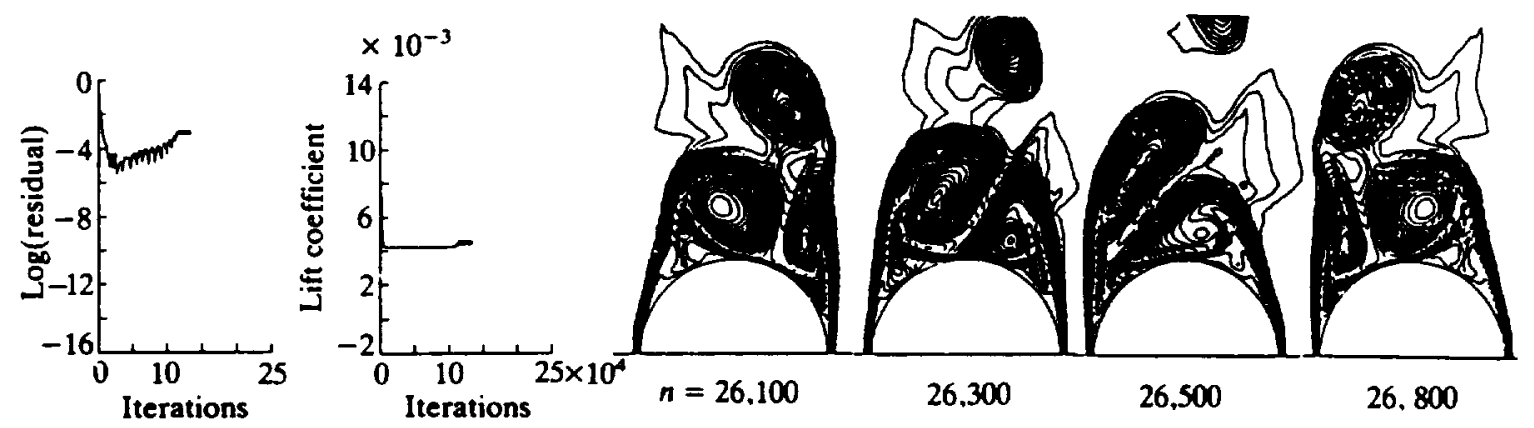

Full Navier-Stokes eqs. (FDS)

Figure 4. Comparison of unsteady asymmetric flow solutions with vortex shedding around a circular cone; periodic flow response; $\alpha=30^{\circ}, \mathrm{M}_{\infty}=1 \cdot 8, \operatorname{Re}=10^{5}, \Delta t=10^{-3}$. 
10,000 iterations and the solution was monitored every 500 iterations. The solution showed that the asymmetry was changing from the left side to the right side, which indicated a possibility of unsteady asymmetric vortex shedding. The residual error was also oscillating. The computations were repeated starting from the 3,500 iteration step using time-accurate calculations with $\Delta t=10^{-3}$. The residual-error and lift-coefficient curves show the time history of the solution. It is seen that the residual error and the lift coefficient show a transient response which is followed by a periodic response. Snapshots of the total-pressure-loss contours are shown at time steps of $n=15,000$; 15,$200 ; 15,400$ and 15,700 . At $n=15,000$, the asymmetric flow is seen with an already shed vortex from the right side. As time progresses, the shed vortex is convected in the flow and the primary vortex on the left side stretches upwards, while the primary vortex on the right gets stronger and expands to the left side. At $n=15,700$, the primary vortex from the left side is shed in the flow field. It should be noticed that the solution at $n=15,700$ is exactly a mirror image of that at $n=15,000$. The solution from 15,000-15,700 represents one half the cycle of shedding. The solution from $15,700-16,400$ represents the second one half of the cycle (not shown). The periodicity of the shedding motion is conclusively captured. The period of oscillation is $10^{-3} \times 1,400$ steps $=1.4$ which corresponds to a shedding frequency of 4.488 .

Figure 4 also shows the results of the FVS scheme with the thin-layer NS equations for one-half cycle of oscillation. Using the FVS scheme, the flux limiters are turned on initially and, as can be seen from the logarithmic-residual curve, the solution becomes symmetric and steady after 5,000 time steps. Next, the flux limiters are turned off, and the solution shows a transient response up to 12,000 time steps. Thereafter, the solution becomes periodic with periodic asymmetric vortex shedding. The solution was monitored every 100 time steps, and we show snapshots of the total-pressure-loss contours between $n=13,900$ and $n=14,600$. Although the process of adjusting the time instants in order to match those of the FDS solution is difficult, it is seen that the captured snapshots of the FVS solution almost match those of the FDS solution. Comparing the FVS solutions at $n=13,900$ and 14,600 , it is seen that they are mirror images of each other. Hence, periodic flow response has been achieved with a period of $1,400 \times 10^{-3}=1.4$; which is exactly the same period of shedding as that of the FDS solution. This pinpoints the high numerical dissipation effect of the FVS scheme when the flux limiters are also turned on. The resulting numerical dissipation in the FVS scheme is large enough to dampen the random disturbances of the flow solution. By turning off the flux limiters in the FVS scheme, the random disturbances grow, producing the asymmetric unsteady vortex shedding. This also shows that the FDS scheme, even with the flux limiters turned on, is less dissipative than the FVS scheme. Finally, we show the results of the FDS-scheme with the full NS equations. The solution conclusively confirms the previous solutions and hence the unsteady solution is not scheme- or equation-dependent.

Figure 5 shows snapshots of the surface-pressure coefficient using the FDS and FVS schemes with the thin-layer NS equations. They are in excellent agreement with each other. The oscillation of the side force is clearly seen in this figure.

\subsection{Unsteady Asymmetric Flow Around an Elliptic Section Cone, $f_{r}=0.8$}

Figure 6 shows the solution for an elliptic-section cone with fineness ratio of $0 \cdot 8$. The residual-error curve shows that the solution produces a symmetric flow through the first 5,000 time steps. Afterwards, the solution shows a transient unsteady flow response for 2,500 time steps which is followed by an unsteady, perfectly periodic, vortex-shedding 

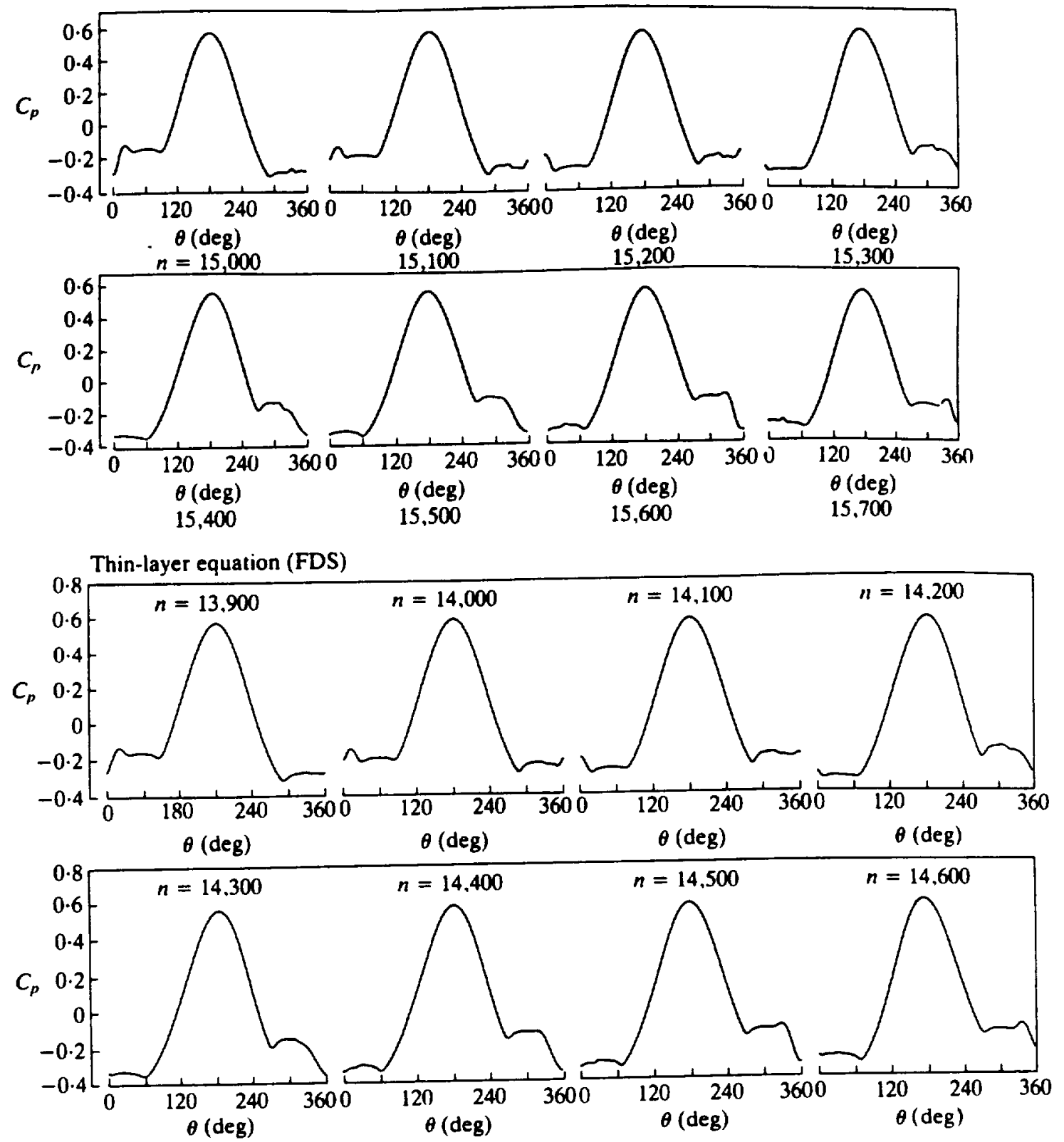

Thin-layer equation (FVS)

Figure 5. Comparison of snapshots of surface-pressure coefficients around a circular cone; $\alpha=30^{\circ}$, $\mathrm{M}_{\infty}=1 \cdot 8, \operatorname{Re}=10^{5}, \Delta t=10^{-3}$.

solution. The lift-coefficient curve shows the same nature of the solution as that of the residual-error curve. This case is carried out using time-accurate stepping with $\Delta t=10^{-3}$.

We also show snapshots of the total-pressure-loss contours and surface-pressure coefficients at the time steps of $12,000,12,500,13,000,13,500,14,000$ and 14,500 . The solutions at $n=12,000$ and 14,500 are mirror images of each other which confirm that the solution is periodic. The period of oscillation is $5,000 \times 10^{-3}=5$ which corresponds to a shedding frequency of 1.257. At $n=12,000$, the total-pressure-loss contours show that the right-side vortex is stretched, having two vortices; one at the top and the second one below it. In addition, a secondary vortex is seen at the surface. The left-side vortex has expanded to the right with two vortices beneath it. At $n=12,500$, 

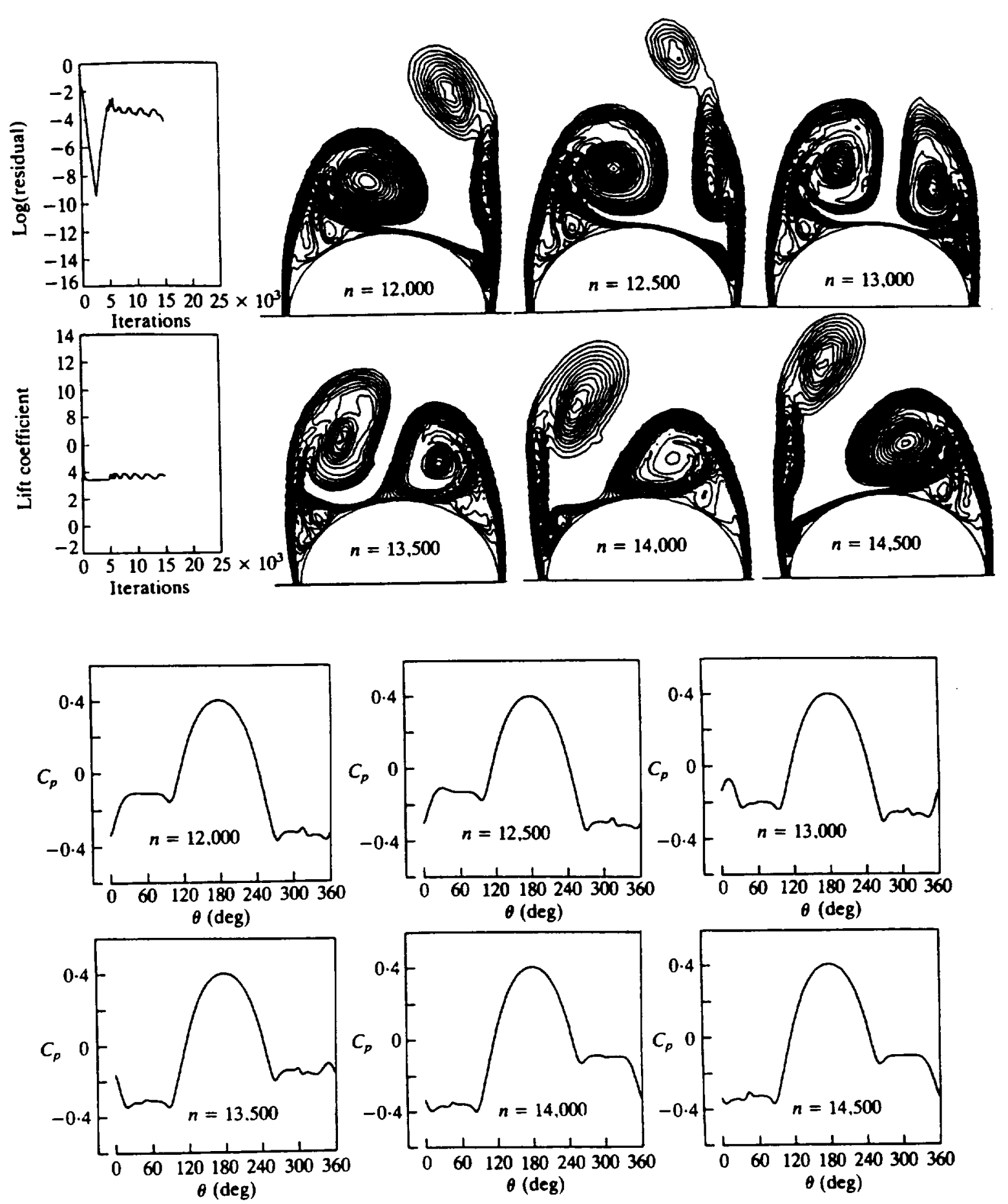

Figure 6. Unsteady asymmetric flow solution with vortex shedding around an elliptic-section cone; periodic flow response; $\alpha=25^{\circ}, \mathrm{M}_{\infty}=1 \cdot 5, \operatorname{Re}=10^{5}, f_{r}=0 \cdot 8, \Delta t=10^{-3}$.

the top vortex on the right side has been almost shed while the one below it is expanding. At $n=13,000$, the top vortex on the right side has been shed and convected with the flow, while the vortex below it is expanding to the left. As time passes, the vortex on the left side is stretching upwards and the vortex on the right side is expanding to the left, as seen from the snapshots at $13,000,13,500$ and 14,000 . At $n=14,500$, the vortices on the left side and right side become mirror images of those on the right side and left side, respectively, at $n=12,000$. Again, this case conclusively shows that unsteady vortex-shedding flows are captured. 
5.5. Unsteady Asymmetric Flow Around an Elliptic-Section Cone, $f_{r}=\mathbf{0} \cdot 2$ (Thick Wing Case)

This case is presented to show that asymmetric vortex shedding also exists for wing-like sections. Here, the elliptic-section fineness ratio is reduced to $\mathbf{0 . 2}$. To obtain this impressive flow case, we have to decrease the free-stream Mach number to 1.4 , increase the angle of attack to $34^{\circ}$ and increase the free-stream Reynolds number to $2 \times 10^{6}$. These adjustments have been made to enhance the critical flow conditions for flow asymmetry. For the same section fineness ratio, same angle of attack and same free-stream Mach number and for free-stream Reynolds number range of $10^{5}$ $1.5 \times 10^{6}$, the flow is either symmetric or steady asymmetric. It is unsteady only when the Reynolds number is increased to $2 \times 10^{6}$.
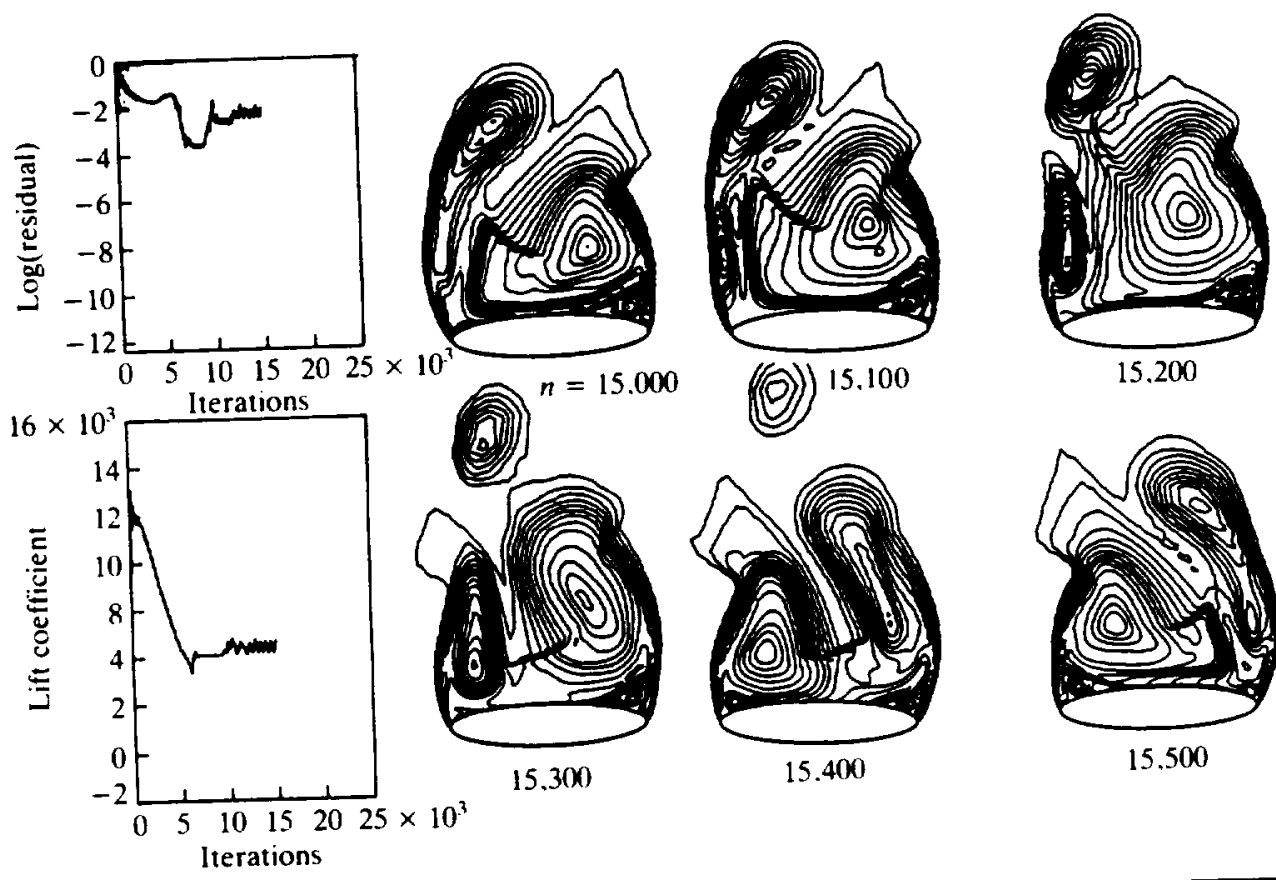

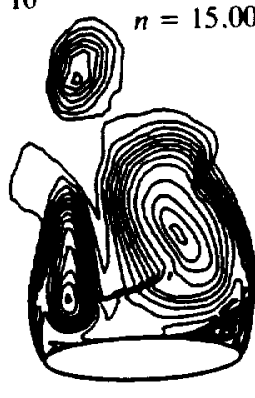

15,300
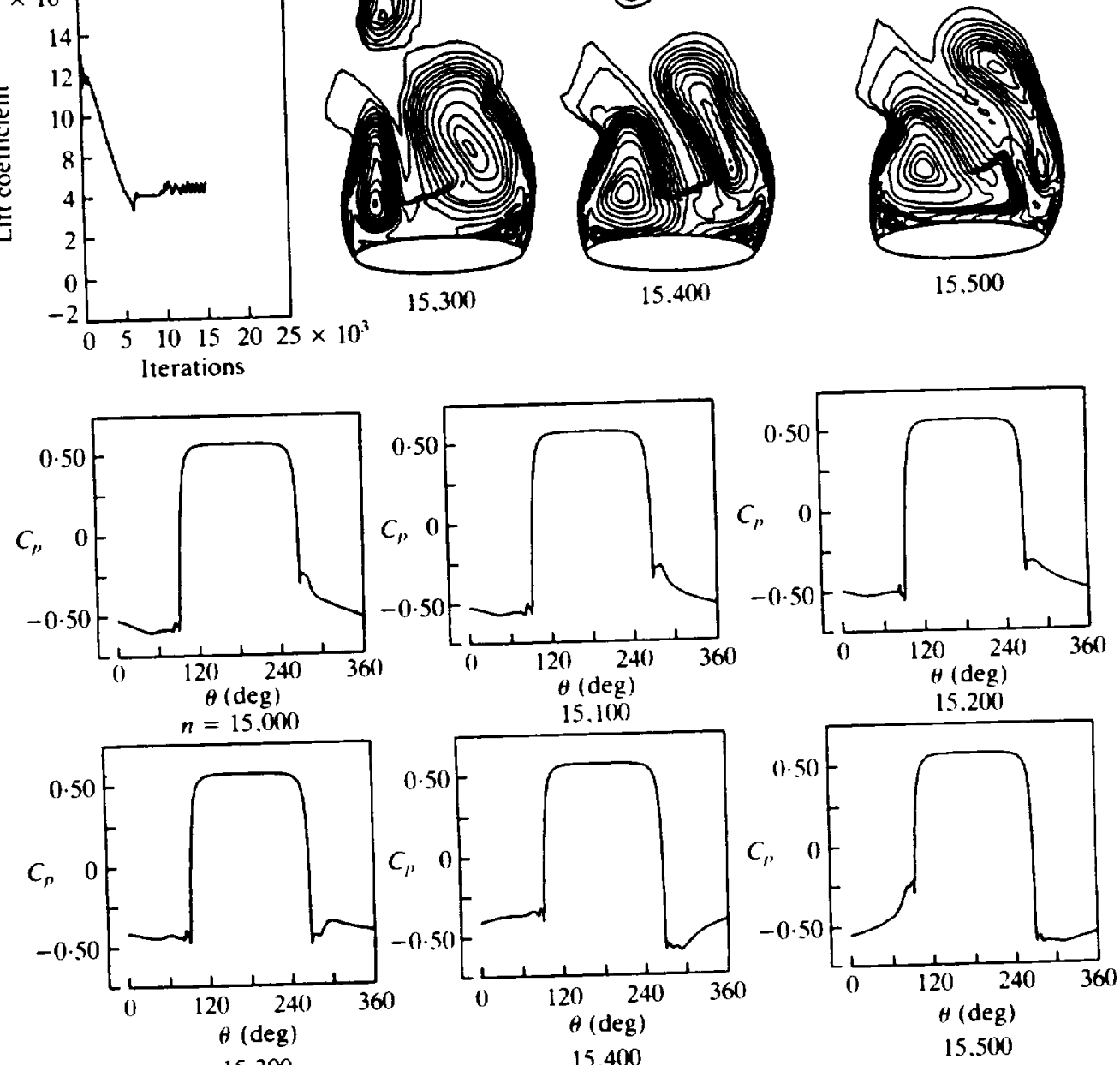

15.300

15.400

15.500

Figure 7. Unsteady asymmetric flow solution with vortex shedding around an elliptic-section cone (a thick wing); periodic flow response; $\alpha=34^{\circ}, M_{\infty}=1 \cdot 4, \operatorname{Re}=2 \times 10^{6}, f_{r}=0.2, \Delta t=2 \times 10^{-3}$. 
Figure 7 shows the time-accurate $\left(\Delta t=2 \times 10^{-3}\right)$ results of this case which includes the time-history of residual error, the time-history of the lift coefficient, snap shots of the total-pressure-loss contours and snapshots of the surface-pressure coefficients. The snapshots at $n=15,000,15,100,15,200,15,300,15,400$ and 15,500 represent approximately one-half the cycle of the periodic flow response. The total-pressure-loss contour at $n=15,000$ shows that the left-side vortex is stretched, while the right-side vortex has expanded, covering a large region of the left side of the flow domain over the wing. Under the right-side vortex, a strong secondary vortex is formed. At $n=15,100$, the left-side vortex shows two regions of vortical flows; one at the top and another one below it. Both vortex regions of the left vortex rotate in the same clockwise direction. At $n=15,200$, the top vortex is shed into the flow field, while the one below it gets stronger and stretches upwards. At $n=15,300$ and 15,400 , the shed vortex from the left side is convected in the flow, the left vortex is expanding to the right and convecting vorticity to the right vortex. The right vortex is getting stronger, shrinking in thickness and stretching upwards. A secondary vortex is forming under the left vortex, and the secondary vortex under the right vortex is diminishing, at $n=15,100$, the flow is approximately a mirror image of that at $n=15,500$. The number of time steps for one cycle of periodic response is 1050 , which gives a period of oscillation of $2 \times 10^{-3} \times 1,050=2 \cdot 1$ corresponding to a frequency of 2.992 .

\subsection{Unsteady Asymmetric Flow Around a Diamond-Section Cone, $f_{4}=0.2$ (Thick Wing CASE)}

Figure 8 shows the results of the time-accurate $\left(\Delta t=5 \times 10^{-4}\right)$ results for this case which include snapshots of the total-pressure-loss contours and snapshots of the surface-pressure coefficients. The snapshots are given at $n=11,500,12,000,12,500$, $13,000,13,500$ and 14,000 . The number of time steps for one cycle of periodic response is 4,500 , which gives a period of oscillation of $5 \times 10^{-4} \times 4,500=2 \cdot 25$ corresponding to a frequency of 2.793 . It should be noted here that the angle of attack is $38^{\circ}$, which is higher than that of the elliptic-section cone of Figure 7, where the angle of attack is $34^{\circ}$ and all the other flow conditions are the same. Comparing the surface pressure curves of the elliptic-section wing (Figure 7) and the diamond-section wing (Figure 8), we conclude that the diamond-section wing has less asymmetric strength and higher lift coefficient than those of the elliptic-section wing.

\section{CONCLUDING REMARKS}

The unsteady, compressible, thin-layer, Navier-Stokes equations are used along with several computational schemes to numerically simulate steady and unsteady asymmetric vortex flows around cones. The concept of the locally conical flow assumption has been developed and discussed. A steady asymmetric flow solutions has been presented and verified for a circular cone. Passive control of the flow asymmetry has been demonstrated for the circular cone by using side strakes. Unsteady, asymmetric vortex flows with periodic vortex shedding have been presented for cones with a circular section, an elliptic section of fineness ratio of 0.8 , an elliptic section of fineness ratio of $\mathbf{0 . 2}$ and a diamond section of fineness ratio of $\mathbf{0 . 2}$. The unsteady asymmetric flow solution for the circular cone has been verified using two schemes with the thin-layer and full NS equations. The present study shows that, for the same flow conditions, circular section cones produce the strongest flow asymmetry while the diamond section cones produce the weakest flow asymmetry. It is conclusive that unsteady flow asymmetry with vortex shedding has been captured. It should be noted 

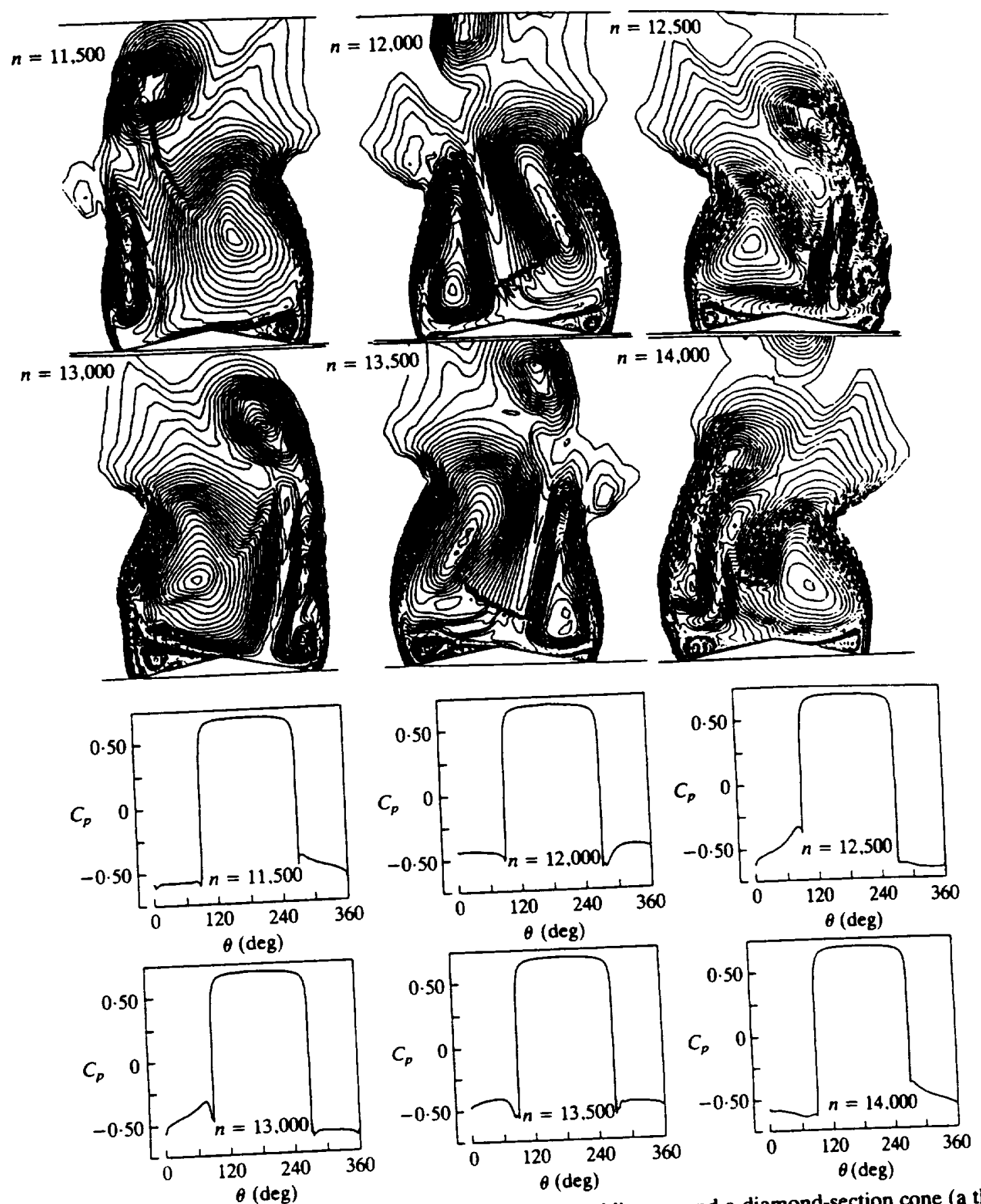
$\theta(\mathrm{deg})$
Figure 8. Unsteady asymmetric flow solution with vortex shedding around a diamond-section cone

that these solutions are based on the locally conical flow assumption and hence they must not be used for quantitative comparisons with three-dimensional flow results. The reason behind such a restriction is the length scale involved with the unsteady and viscous terms of the locally conical NS equations. However, the solutions are computationally economical for qualitative and parametric studies of asymmetric flows.

\section{ACKNOWLEDGEMENT}

This research work is supported by the NASA Langley Research Center under Grants No. NAS1-18584-71 and NAG-1-994. 


\section{REFERENCES}

FIDDES, S. P. 1989 Recent developments in the production of separated flow past slender bodies at incidence. In Proceedings Royal Aeronautical Society Conferences on Prediction and Exploitation of Separated Flows, pp. 31.1-31.17, London, U.K.

Kandil, O. A. \& ChuANG, H. A. 1989 Unsteady Navier-Stokes computations past oscillating delta wings at high incidence. AIAA Paper No. 89-081. Also AIAA Journal 28, 1565-1572.

Kandil, O. A., Wong, T.-C. \& LiU, C. H. 1990a. Prediction of steady and unsteady asymmetric flows around cones. AIAA Paper No. 90-0598. flows around cones. AIAA Paper No. $90-1990 \mathrm{~b}$ Asymmetric supersonic flow around cones
KandiL, O. A., Wong, T.-C. \& LiU, C. H. 190 ,
with noncircular sections. AGARD Conference Proceedings No. 493, Missile Aerodynamics, pp. 16.1-16.11.

KeENER, E. R. \& Chapman, G. R. 1977 Similarity in vortex asymmetries over slender bodies and wings. AlAA Journal 15, 1370-1372.

LAMONT, P. J. 1980 Pressure around an inclined ogive cylinder with laminar, transitional and turbulent separation. AIAA Journal 20, 1492-1499.

LAMONT, P. R. 1982 The complex asymmetric flow over a 3.5D ogive nose and cylindrical afterbody at high angles of attack. AIAA Paper No. 82-0053.

Marconi, F. 1988 Asymmetric flows about sharp cones in a supersonic stream. In Proceedings of 11th International Conference on Numerical Methods in Fluid Dynamics, Williamsburg,

VA, U.S.A.
Moskovitz, C., HALL, R. \& Dejarnette, F. 1990 Experimental investigation of a new device to control asymmetric flowfield on forebodies at large angles of attack. AIAA Paper No. 90-0069.

No, T. T. 1989 On leading edge vortex and its control. AIAA Paper no. 89-3346-CP.

NG, T. T. 1990 Aerodynamic control of NASP-type vehicles through vortex manipulation. AIAA Paper No. 90-0594.

Peake, D. J., Owen, F. K. \& Higuch, H. 1979 Symmetrical and asymmetrical separations about a yawed cone. $A G A R D C P-247,16.1-16.27$.

PEAke, D. J. \& TOBAK, M. 1982 Three-dimensional flows about simple components at angle of attack. AGARD LS-121, High angle-of-attack aerodynamics, 2.1-2.56.

Rediniotis, O., Stapountzis, H. \& Telionis, D. P. 1988 Vortex shedding over nonparallel edges. VPI \& SU Engineering Report, VPI-88-39.

RumseY, L. C. \& ANDERson, W. K. 1988 Some numerical aspects of unsteady Navier-Stokes computations over airfoils using dynamic meshes. AIAA Paper No. 88-0329.

Low subsonic measurements of static and dynamic stability derivatives of six HANKS, R. E. 1963 Low suleading-edge sweep angles of $70^{\circ}-84^{\circ}$. NASA TND-1822.

flat plate wings MARCONI, F. 1989 Computations of Navier-Stokes solutions exhibiting asymmetric vortices. AIAA Paper No. 89-1817.

Skow, A. M. \& PeAKe, D. J. 1982 Control of forebody vortex orientation by asymmetric air injection (Part B)-Details of the flow structure. AGARD LS-121, High angle-of-attack aerodynamics, 10.1-10.22.

STAHL, W. 1989 Suppression of asymmetry of vortex flow behind a circular cone at high incidence. AIAA Paper No. 89-3372-CP.

Travella, D. A., Schiff, L. B. \& Cummings 1990 Pneumatic vortical flow control at high angles of attack. AlAA Paper No. 90-0098. 